\title{
Heat content and inradius for regions with a Brownian boundary
}

\author{
M. van den Berg ${ }^{1}$ \\ E. Bolthausen ${ }^{2}$ \\ F. den Hollander ${ }^{3}$
}

12 March 2013

\begin{abstract}
In this paper we consider $\beta[0, s]$, Brownian motion of time length $s>0$, in $m$-dimensional Euclidean space $\mathbb{R}^{m}$ and on the $m$-dimensional torus $\mathbb{T}^{m}$. We compute the expectation of (i) the heat content at time $t$ of $\mathbb{R}^{m} \backslash \beta[0, s]$ for fixed $s$ and $m=2,3$ in the limit $t \downarrow 0$, when $\beta[0, s]$ is kept at temperature 1 for all $t>0$ and $\mathbb{R}^{m} \backslash \beta[0, s]$ has initial temperature 0 , and (ii) the inradius of $\mathbb{T}^{m} \backslash \beta[0, s]$ for $m=2,3, \cdots$ in the limit $s \rightarrow \infty$.
\end{abstract}

AMS 2000 subject classifications. 35J20, 60G50.

Key words and phrases. Laplacian, Brownian motion, Wiener sausage, heat content, inradius, spectrum.

Acknowledgment. MvdB was supported by The Leverhulme Trust, Research Fellowship 2008/0368, EB by SNSF-grant 20-100536/1, and FdH by ERC Advanced Grant 267356-VARIS.

\footnotetext{
${ }^{1}$ School of Mathematics, University of Bristol, University Walk, Bristol BS8 1TW, United Kingdom. ${ }^{2}$ Institut für Mathematik, Universität Zürich, Winterthurerstrasse 190, CH-8057 Zürich, Switzerland.

${ }^{3}$ Mathematical Institute, Leiden University, P.O. Box 9512, 2300 RA Leiden, The Netherlands.
} 


\section{Introduction and main results}

Asymptotic properties of the heat content and the inradius for regions with a fractal boundary have received a lot of attention in the literature. Most of the focus has been on porous regions (e.g. the $m$-dimensional Euclidean space $\mathbb{R}^{m}$ from which a Poisson cloud of non-polar sets is removed [20]), and regions with a fractal polygonal boundary e.g. the von Koch snow flake and its relatives [2, 3, 6]. In this paper we consider the region obtained from $\mathbb{R}^{m}$ or the $m$-dimensional torus $\mathbb{T}^{m}$ by cutting out a Brownian path of time length $s$. In Sections 1.1 and 1.2 we consider the heat content, and in Section [1.3 the inradius. We formulate some open problems in Section 1.4 The proofs are deferred to Sections 23

\subsection{Heat content outside compact sets}

Let $K$ be a compact non-polar set in $\mathbb{R}^{m}$ with boundary $\partial K$, and let $v: \mathbb{R}^{m} \backslash K \times[0, \infty) \rightarrow \mathbb{R}$ be the unique weak solution of the heat equation

$$
\left\{\begin{array}{llll}
\Delta v(x ; t) & =\partial v(x ; t) / \partial t, & & x \in \mathbb{R}^{m} \backslash K, t>0, \\
v(x ; t) & =1, & & x \in \partial K, t>0, \\
v(x ; 0) & =0, & & x \in \mathbb{R}^{m} \backslash K .
\end{array}\right.
$$

Then $v(x ; t)$ represents the temperature at point $x$ at time $t$ when $\partial K$ is kept at temperature 1 and the initial temperature is 0 . The heat content of $\mathbb{R}^{m} \backslash K$ at time $t$ is defined by

$$
E_{K}(t)=\int_{\mathbb{R}^{m} \backslash K} v(x ; t) d x .
$$

If $\partial K$ is $C^{\infty}$, then $E_{K}(t)$ has an asymptotic series expansion for $t \downarrow 0$ of the form

$$
E_{K}(t)=\sum_{j=1}^{J} a_{j}(K) t^{j / 2}+O\left(t^{(J+1) / 2}\right), \quad t \downarrow 0, J \in \mathbb{N},
$$

where the coefficients are local geometric invariants of $\mathbb{R}^{m} \backslash K$. In particular,

$$
\begin{aligned}
& a_{1}(K)=2 \pi^{-1 / 2} \int_{\partial K} d z, \\
& a_{2}(K)=2^{-1}(m-1) \int_{\partial K} H(z) d z,
\end{aligned}
$$

where $d z$ is the surface measure on $\partial K$, and $H(z)$ is the mean curvature at $z$ of $\partial K$ with inward orientation. Formulas of this type can be found in the general setting of Riemannian manifolds and Laplace-type operators [11. The case where $\partial K$ is only $C^{3}$ was settled by probabilistic tools in [7, and the expansion in (1.3) holds for $J=2$.

The asymptotic behaviour for $t \rightarrow \infty$ is different and its analysis does not require smoothness of $\partial K$. For $m=3$ it is shown in [12, [16] (see [18, for earlier results) that if $K$ is a compact set, then

$$
E_{K}(t)=\sum_{j=1}^{3} b_{j}(K) t^{(3-j) / 2}+O\left(t^{-1 / 2}\right), \quad t \rightarrow \infty,
$$

with

$$
\begin{aligned}
& b_{1}(K)=\operatorname{cap}(K), \\
& b_{2}(K)=2^{-1} \pi^{-3 / 2} \operatorname{cap}(K)^{2}, \\
& b_{3}(K)=(4 \pi)^{-2} \operatorname{cap}(K)^{3}-|K|-(8 \pi)^{-1} \int_{K} \int_{K}\|x-y\| \mu_{K}(d x) \mu_{K}(d y),
\end{aligned}
$$


where $\|\cdot\|$ is the Euclidean norm, $\mu_{K}$ is the equilibrium measure of $K, \operatorname{cap}(K)=\int_{K} \mu_{K}(d x)$ is the Newtonian capacity of $K$, and $|K|$ is the Lebesgue measure of $K$. For $m=2$ it is shown in [13] that if $K$ is a non-polar set, then

$$
E_{K}(t)=t \sum_{j=1}^{J} b_{j}(K)(\log t)^{-j}+O\left(t(\log t)^{-(J+1)}\right), \quad t \rightarrow \infty, J \in \mathbb{N}
$$

where $b_{1}(K)=4 \pi$, and where the higher-order coefficients all depend on the logarithmic capacity of $K$ only.

For a wide class of regions with a fractal boundary it is known that $E_{K}(t)$ is comparable with $t^{(m-d) / 2}$ for $t \downarrow 0$, where $d$ is the interior Minkowski dimension of the boundary of $\mathbb{R}^{m} \backslash K[2$. In the case of a self-similar boundary it is sometimes possible to obtain more detailed results [6], 3]. It turns out that if there is a dominant arithmetic sequence of length scales, then the leading term is $t^{(m-d) / 2}$ times a periodic function of $\log \left(t^{-1}\right)$. If there is no such sequence, then the leading term is $t^{(m-d) / 2}$ times a constant. However, neither the periodic function nor the constant is known explicitly. In Section 1.2 below we study the case where $K$ is a Brownian path of time length $s$. We will see that for this case there is no periodic function of $\log \left(t^{-1}\right)$.

\subsection{Expectation of the heat content outside a Brownian path}

The solution of (1.1) is given by

$$
v(x ; t)=\mathbb{P}_{x}\left(\tau_{K} \leq t\right),
$$

where $\tau_{K}=\inf \{u \geq 0: \bar{\beta}(u) \in K\}$ with $\left(\bar{\beta}(u), u \geq 0 ; \mathbb{P}_{x}, x \in \mathbb{R}^{m}\right)$ Brownian motion on $\mathbb{R}^{m}$. Let $E_{\bar{\beta}[0, s]}(t)$ denote the heat content of $\mathbb{R}^{m} \backslash \beta[0, s]$ at time $t$, and let

$$
E(s, t)=\mathbb{E}_{0}\left(E_{\beta[0, s]}(t)\right)
$$

denote its expectation. Since $\operatorname{cap}(\beta[0, s])=0$ for $m=4,5, \cdots$, only $m=2,3$ are relevant. In Section 2 we will prove the following duality property.

Theorem 1.1 (a) Let $m=2,3$. Then for $s, t>0$,

$$
E(s, t)=E(t, s), \quad E(s, t)=(t / s)^{m / 2} E\left(s, s^{2} / t\right) .
$$

(b) Let $m=3$. Then for $s>0$,

$$
E(s, t)=c_{1}(s) t^{1 / 2}+c_{2}(s) t+c_{3}(s) t^{3 / 2}+O\left(t^{2}\right), \quad t \downarrow 0,
$$

with

$$
\begin{aligned}
& c_{1}(s)=\mathcal{C}_{1} s, \\
& c_{2}(s)=2^{-1} \pi^{-3 / 2} \mathcal{C}_{2} s^{1 / 2}, \\
& c_{3}(s)=(4 \pi)^{-2} \mathcal{C}_{3}-(8 \pi)^{-1} \mathbb{E}_{0}\left(\int_{\mathbb{R}^{3}} \int_{\mathbb{R}^{3}}\|x-y\| \mu_{\beta[0,1]}(d x) \mu_{\beta[0,1]}(d y)\right),
\end{aligned}
$$

where

$$
\mathcal{C}_{i}=\mathbb{E}_{0}\left((\operatorname{cap}(\beta[0,1]))^{i}\right), \quad i=1,2,3 .
$$

(c) Let $m=2$. Then for $s>0$,

$$
E(s, t)=4 \pi s\left(\log \left(t^{-1}\right)\right)^{-1}+O\left(\left(\log \left(t^{-1}\right)\right)^{-2}\right), \quad t \downarrow 0 .
$$

Theorem 1.1(a) states a duality property from which Theorem 1.1(b-c) easily follows. Indeed from (1.4 1.5) and (1.8) we obtain that for $m=3$,

$$
E(s, t)=\sum_{j=1}^{3} c_{j}(s) t^{(3-j) / 2}+O\left(t^{-1 / 2}\right), \quad t \rightarrow \infty
$$


with $c_{j}(s)=\mathbb{E}_{0}^{1}\left(b_{j}(\beta[0, s])\right)$. By combining (1.12) with (1.9), we obtain the claim in Theorem 1.1(b). Similarly, it follows from (1.6) that for $m=2$,

$$
E(s, t)=4 \pi t(\log t)^{-1}+O\left(t(\log t)^{-2}\right), \quad t \rightarrow \infty .
$$

By combining (1.13) with (1.9), we obtain the claim in Theorem 1.1(c).

\subsection{Expectation of the inradius of a torus cut by a Brownian path}

Let $\mathbb{T}^{m}$ denote the $m$-dimensional torus, which we identify with the set $\left(-\frac{1}{2},+\frac{1}{2}\right]^{m}$. We denote the distance on $\mathbb{T}^{m}$ between points $x$ and $y$ by $d(x, y) . \quad \mathbb{T}^{m}$ is a compact and connected Riemannian manifold without boundary. The associated Laplace-Beltrami operator is the generator of Brownian motion on $\mathbb{T}^{m}$. The latter process can be obtained by wrapping Brownian motion on $\mathbb{R}^{m}$ around $\mathbb{T}^{m}$. Namely, let $\left(\tilde{\beta}(u), u \geq 0 ; \mathbb{P}_{x}, x \in \mathbb{R}^{m}\right)$ denote Brownian motion on $\mathbb{R}^{m}$, with the Laplacian as generator, and put

$$
\beta(u)=\left(\tilde{\beta}(u)+\left(\frac{1}{2}, \cdots, \frac{1}{2}\right)\right)\left(\bmod \mathbb{Z}^{m}\right)-\left(\frac{1}{2}, \cdots, \frac{1}{2}\right) .
$$

Then $\left(\beta(u), u \geq 0 ; \mathbb{P}_{x}, x \in \mathbb{R}^{m}\right)$ is Brownian motion on $\mathbb{T}^{m}$.

On $\mathbb{T}^{m}$ we define a random distance function $d_{s}$ by putting

$$
d_{s}(x)=\inf \{d(x, y): y \in \beta[0, s]\}, \quad x \in \mathbb{T}^{m},
$$

i.e., the distance of $x$ to $\beta[0, s]$. The inradius of $\mathbb{T}^{m} \backslash \beta[0, s]$ is the random variable $\rho(s)$ defined by

$$
\rho(s)=\sup \left\{d_{s}(x): x \in \mathbb{T}^{m}\right\} .
$$

The supremum is attained because $d_{s}$ is continuous and $\mathbb{T}^{m}$ is compact. Hence there exists an open ball with radius $\rho(s)$ in $\mathbb{T}^{m} \backslash \beta[0, s]$. The inradius is a non-trivial random variable in all dimensions.

Theorem 1.2 If $m=3,4, \cdots$, then

$$
\lim _{s \rightarrow \infty}\left(\frac{s}{\log s}\right)^{1 /(m-2)} \mathbb{E}_{0}(\rho(s))=\left(\frac{m}{(m-2) \kappa_{m}}\right)^{1 /(m-2)}
$$

where $\kappa_{m}$ is the Newtonian capacity of the ball with radius 1 in $\mathbb{R}^{m}$, given by

$$
\kappa_{m}=4 \pi^{m / 2}[\Gamma((m-2) / 2)]^{-1} .
$$

Theorem 1.3 If $m=2$, then

$$
\lim _{s \rightarrow \infty} s^{-1 / 2} \log \mathbb{E}_{0}(\rho(s))=-\pi^{1 / 2} .
$$

\subsection{Discussion and open problems}

Heat content. Since the Minkowski dimension of the Brownian path equals $d=2$, the power $1 / 2$ in (1.10) for $m=3$ agrees with the exponent $(m-d) / 2$ mentioned below (1.6). The absence of a periodic function multiplying the term $t^{1 / 2}$ reflects the fact that there is no dominant arithmetic sequence of length scales in the Brownian path. Comparing (1.10) with (1.4 1.5), we see that $\beta[0, s]$ has an effective area $2^{-1} \pi^{1 / 2} \mathcal{C}_{1} s$ and an effective mean curvature integral $2^{-1} \pi^{-3 / 2} \mathcal{C}_{2} s^{1 / 2}$.

We see from Theorem 1.1 that the complement of the Brownian path heats up much faster for $m=2$ than for $m=3$ as $t$ increases from 0 . With the help of (1.6) it is actually possible to obtain the full asymptotic series for $m=2$. The geometry of $\beta[0,1]$ enters into this series only via the expectation of the powers of the logarithm of the logarithmic capacity of $\beta[0,1]$.

Inradius. Theorems 1.21 .3 identify the scaling behavior of the expectation of the inradius. Strong laws of large numbers were derived in [9] and [10]. Our proofs for the upper bounds in Section 3.1 are 
based on Wiener sausage estimates and spectral decomposition, while the lower bounds in Section 3.2 and Section 3.3 respectively, are based on results of [9] and [10] which rely in turn on excursions of Brownian motions.

Spectrum. There are several other set functions that are closely related to the inradius such as the principal Dirichlet eigenvalue. For $s>0$, let $\Delta_{s}$ be the Laplacian acting in $L^{2}\left(\mathbb{T}^{m} \backslash \beta[0, s]\right)$ with Dirichlet boundary condition on $\beta[0, s]$. The spectrum of $-\Delta_{s}$ is bounded from below by the spectrum of $-\Delta$ on $\mathbb{T}^{m}$. Since the latter is discrete, the spectrum of $-\Delta_{s}$ is discrete, with eigenvalues $\left(\lambda_{j, s}\right)_{j \in \mathbb{N}}$, labeled in non-decreasing order and including multiplicities. Since the Newtonian capacity of $\beta[0, s]$ is zero for $m>3$, the spectrum of $-\Delta_{s}$ is non-trivial if and only if $m=2,3$. Since $s \mapsto \mathbb{T}^{m} \backslash \beta[0, s]$ is decreasing we have, by domain monotonicity of Dirichlet eigenvalues [17, that $s \mapsto \lambda_{j, s}$ is increasing.

Conjecture 1.4 If $m=2$, then

$$
\lim _{s \rightarrow \infty} s^{-1 / 2} \log \mathbb{E}_{0}\left(\lambda_{1, s}\right)=2 \pi^{1 / 2} .
$$

Conjecture 1.5 If $m=3$, then

$$
\lim _{s \rightarrow \infty}\left(\frac{\log s}{s}\right)^{2} \mathbb{E}_{0}\left(\lambda_{1, s}\right)=9^{-1}(2 \pi)^{4} .
$$

The heuristic behind Conjecture 1.4 is as follows. The complement of the Brownian path $\beta[0, s]$ in $\mathbb{T}^{2}$ consists of simply connected open components. Hence the spectrum of the Dirichlet Laplacian acting in $L^{2}\left(\mathbb{T}^{2} \backslash \beta[0, s]\right)$ is the union of the spectra of the Dirichlet Laplacian for all the components. In particular, $\lambda_{1, s}$ is the minimum over all first eigenvalues of these components. For a simply connected open set $\Omega$ in $\mathbb{R}^{2}$ with inradius $\rho$ we know from 1 that the first Dirichlet eigenvalue is comparable to $\rho^{-2}$. The same is not true for the bottom of the spectrum of a simply connected open set $\Omega$ in $\mathbb{T}^{2}$. Indeed, the inradius of $\mathbb{T}^{2}$ is bounded from above by half its diameter. Hence, if $\Omega$ is almost all of $\mathbb{T}^{2}$, then the bottom of the spectrum is close to 0 while $\rho^{-2}$ is bounded away from 0 . However, for large $s$ it is very unlikely that such a large component exists. Thus, we have that the typical component for large $s$ has small $\rho(s)$, and hence, by [1],

$$
\lambda_{1, s} \asymp \rho(s)^{-2} .
$$

The right-hand side is of order $e^{2(\pi s)^{1 / 2}}$, which explains (1.14).

The heuristic behind Conjecture 1.5 is as follows. The simplifying features for $m=2$ are absent for $m=3$. We expect that with high probability the largest open ball in $\mathbb{T}^{3} \backslash \beta[0, s]$ with inradius $\rho(s)$ is "densely surrounded" by the Brownian path. So $\lambda_{1, s}$ is the first Dirichlet eigenvalue of a ball with radius $\rho(s)$ in $\mathbb{T}^{3}\left(\right.$ or $\left.\mathbb{R}^{3}\right)$. Hence

$$
\lambda_{1, s} \approx \pi^{2} \rho(s)^{-2} .
$$

We also expect that the inradius $\rho(s)$ gets very narrowly distributed around its mean as $s \rightarrow \infty$. Hence for large $s$,

$$
\mathbb{E}_{0}\left(\lambda_{1, s}\right) \approx \pi^{2}\left(\mathbb{E}_{0}(\rho(s))\right)^{-2} .
$$

The asymptotic behaviour of the latter expectation can be read off from Theorem 1.2 for $m=3$, and implies (1.15).

Large deviations. As explained at the end of Section 2, it is easy to show that for $m=3$ and $s>0$ the following strong law of large numbers holds:

$$
\lim _{t \downarrow 0} E_{\beta[0, s]}(t) / E(s, t)=1 \quad \mathbb{P}_{0}-\text { a.s. }
$$

We expect that both $\rho(s)$ and $\lambda_{1, s}$ satisfy the strong law of large numbers as $s \rightarrow \infty$. It is interesting to determine their large deviation behaviour. For $m \geq 3$ this was achieved in [14], which was inspired by an unpublished earlier version of the present paper. Interestingly, the large deviations are so uncostly in one direction that they do not imply our results about the expectation. 


\section{Proof of Theorem 1.1}

Let

$$
\left(\beta_{i}(u), u \geq 0 ; \mathbb{P}_{x}^{i}, x \in \mathbb{R}^{m}\right), \quad i=1,2,
$$

be two independent Brownian motions on $\mathbb{R}^{m}$. Recalling (1.7), we have from (1.2) that

$$
E_{K}(t)+|K|=\mathbb{E}_{0}^{2}\left(\left|W_{\beta_{2}}^{K}(t)\right|\right),
$$

where

$$
W_{\beta_{2}}^{K}(t)=\cup_{u \in[0, t]}\left\{K+\beta_{2}(u)\right\}=K+\beta_{2}[0, t]
$$

is the $K$-set Wiener sausage associated with $\beta_{2}$ up to time $t$. Now choose $K=\beta_{1}[0, s], s>0$. Then, since $\left|\beta_{1}[0, s]\right|=0$, the heat content in $\mathbb{R}^{m} \backslash \beta_{1}[0, s]$ becomes

$$
E_{\beta_{1}[0, s]}(t)=\mathbb{E}_{0}^{2}(|W(s, t)|)
$$

with

$$
W(s, t)=W_{\beta_{2}}^{\beta_{1}[0, s]}(t)=\beta_{1}[0, s]+\beta_{2}[0, t]=W_{\beta_{1}}^{\beta_{2}[0, t]}(s) .
$$

The expected heat content becomes

$$
E(s, t)=\mathbb{E}_{0}^{1}\left(E_{\beta_{1}[0, s]}(t)\right)=\left(\mathbb{E}_{0}^{1} \otimes \mathbb{E}_{0}^{2}\right)(|W(s, t)|)=\mathbb{E}_{0}^{2}\left(E_{\beta_{2}[0, t]}(s)\right) .
$$

We have the following two elementary lemmas.

Lemma 2.1 For all $s, t \geq 0$,

$$
E(s, t)=E(t, s)
$$

Proof. Note that

$$
E(s, t)=\left(\mathbb{E}_{0}^{1} \otimes \mathbb{E}_{0}^{2}\right)(|W(s, t)|)=\int_{\mathbb{R}^{m}} d x\left(\mathbb{P}_{x}^{1} \otimes \mathbb{P}_{0}^{2}\right)\left(\tau_{\beta_{2}[0, t]} \leq s\right)
$$

with

$$
\tau_{\beta_{2}[0, t]}=\inf \left\{u \geq 0: \beta_{1}(u) \in \beta_{2}[0, t]\right\} .
$$

We may rewrite (2.1) as

$$
E(s, t)=\int_{\mathbb{R}^{m}} d x\left(\mathbb{P}_{x}^{1} \otimes \mathbb{P}_{0}^{2}\right)\left(\beta_{1}[0, s] \cap \beta_{2}[0, t] \neq \emptyset\right),
$$

from which the symmetry property follows via the change of variable $x \rightarrow-x$.

Lemma 2.2 For all $a>0$ and $s, t \geq 0$,

$$
|W(s, t)| \triangleq a^{-m}\left|W\left(a^{2} s, a^{2} t\right)\right|,
$$

where $\triangleq$ denotes equality in distribution.

Proof. Let $W_{\beta_{1}}^{A}(t)$ is the $A$-set Wiener sausage associated with $\beta_{1}$ up to time $t$, and note the two scaling relations

$$
\beta_{2}[0, t] \triangleq a^{-1} \beta_{2}\left[0, a^{2} t\right], \quad W_{\beta_{1}}^{A}(s) \triangleq a^{-d} W_{\beta_{1}}^{a A}\left(a^{2} s\right) .
$$

The claim follows by choosing $A=a^{-1} \beta_{2}\left[0, a^{2} s\right]$.

Theorem 1.1(a) follows from Lemma 2.1 and Lemma 2.2 with $a^{2}=s / t$. Theorems 1.1(b-c) follow from (1.9), (1.12 1.13) and the scaling relations

$$
\begin{aligned}
\operatorname{cap}\left(\beta_{1}[0, s]\right) & \triangleq s^{1 / 2} \operatorname{cap}\left(\beta_{1}[0,1]\right), \\
\mu_{\beta_{1}[0, s]}(s A) & \triangleq \mu_{\beta_{1}[0,1]}(A),
\end{aligned}
$$


which are valid for any compact subset $A \subset \mathbb{R}^{3}$. The latter imply that $c_{j}(s)=s^{j / 2} c_{j}(1), s>0$, for $m=3$.

A standard renewal argument [18, [19] gives the strong law of large numbers for $|W(s, t)|$, namely, for $s>0$,

$$
\lim _{t \rightarrow \infty}|W(s, t)| / \mathbb{E}_{0}^{1}(|W(s, t)|)=1 \quad\left(\mathbb{P}_{0}^{1} \otimes \mathbb{P}_{0}^{2}\right)-\text { a.s. }
$$

This in turn implies that for $s>0$,

$$
\lim _{t \rightarrow \infty} \mathbb{E}_{0}^{2}(|W(s, t)|) /\left(\mathbb{E}_{0}^{1} \otimes \mathbb{E}_{0}^{2}\right)(|W(s, t)|)=1 \quad \mathbb{P}_{0}^{1}-\text { a.s. }
$$

which is the same as

$$
\lim _{t \rightarrow \infty} E_{\beta_{1}[0, s]}(t) / E(s, t)=1 \quad \mathbb{P}_{0}^{1}-\text { a.s. }
$$

The claim in (1.17) follows from (2.2) via Lemma 2.2 with $a^{2}=s / t$.

\section{Proof of Theorems $1.2-1.3$}

For $x \in \mathbb{T}^{m}$ and $\epsilon>0$, let $T_{x, \epsilon}=\inf \left\{u \geq 0: \beta(u) \in B_{x}(\epsilon)\right\}$, where $\left(\beta(u), u \geq 0 ; \mathbb{P}_{x}, x \in \mathbb{T}^{m}\right)$ is Brownian motion on $\mathbb{T}^{m}$, and $B_{x}(\epsilon)$ is the open ball with center $x$ and radius $\epsilon$ in $\mathbb{T}^{m}$. Then

$$
T_{\epsilon}=\sup _{x \in \mathbb{T}^{m}} T_{x, \epsilon}
$$

is the cover time of $\mathbb{T}^{m}$ by the Wiener sausage with radius $\epsilon$. By translation invariance, we have

$$
\mathbb{P}_{0}\left[T_{\epsilon}>s\right]=\mathbb{P}_{x}\left[T_{\epsilon}>s\right], \quad x \in \mathbb{T}^{m}, s \geq 0,
$$

which, since $\left|\mathbb{T}^{m}\right|=1$, gives

$$
\mathbb{P}_{0}\left[T_{\epsilon}>s\right]=\int_{\mathbb{T}^{m}} d x \mathbb{P}_{x}\left[T_{\epsilon}>s\right]
$$

\subsection{Upper bound}

Let $N \in \mathbb{N}$, and let $\left\{x_{1}, x_{2}, \cdots, x_{N^{m}}\right\}=\left(N^{-1} \mathbb{Z}\right)^{m} \cap \mathbb{T}^{m}$. Let $\eta \in(0,1 / 4)$ be arbitrary, and consider the collection of open balls with centers $\left\{x_{1}, x_{2}, \cdots, x_{N^{m}}\right\}$ and radii $(1-\eta) \epsilon$. There exists $v \in$ $\left\{x_{1}, x_{2}, \cdots, x_{N^{m}}\right\}$ such that $d(x, v) \leq(2 N)^{-1} m^{1 / 2}$. For $N \geq m^{1 / 2} /(2 \epsilon \eta)$, we have $B_{x}(\epsilon) \supset B_{v}((1-\eta) \epsilon)$. This implies that if $N \geq m^{1 / 2} /(2 \epsilon \eta)$, then

$$
\left\{B_{x_{i}}((1-\eta) \epsilon) \cap \beta[0, s] \neq \emptyset, i=1,2, \cdots, N^{m}\right\} \subset\left\{T_{\epsilon} \leq s\right\} .
$$

It follows that

$$
\begin{aligned}
\mathbb{P}_{x}\left[T_{\epsilon}>s\right] & \leq 1-\mathbb{P}_{x}\left[B_{x_{i}}((1-\eta) \epsilon) \cap \beta[0, s] \neq \emptyset, i=1, \cdots, N^{m}\right] \\
& \leq 1-\mathbb{E}_{x}\left[\prod _ { i = 1 } ^ { N ^ { m } } \left(1-1_{\left.\left.B_{x_{i}}((1-\eta) \epsilon) \cap \beta[0, s]=\emptyset\right)\right]}\right.\right. \\
& \leq\left(\sum_{i=1}^{N^{m}} \mathbb{P}_{x}\left[B_{x_{i}}((1-\eta) \epsilon) \cap \beta[0, s]=\emptyset\right]\right) \wedge 1 .
\end{aligned}
$$

By (3.1) and (3.2), we have

$$
\begin{aligned}
\mathbb{P}_{0}\left[T_{\epsilon}>s\right] & \leq \int_{\mathbb{T}^{m}} d x\left(\sum_{i=1}^{N^{m}} \mathbb{P}_{x}\left[B_{x_{i}}((1-\eta) \epsilon) \cap \beta[0, s]=\emptyset\right]\right) \wedge 1 \\
& \leq\left(N^{m} \int_{\mathbb{T}^{m}} d x \mathbb{P}_{x}\left[B_{x_{1}}((1-\eta) \epsilon) \cap \beta[0, s]=\emptyset\right]\right) \wedge 1 .
\end{aligned}
$$


Next, let $\mu_{1,(1-\eta) \epsilon}<\mu_{2,(1-\eta) \epsilon} \leq \cdots$ be the spectrum of the Dirichlet Laplacian acting in $L^{2}\left(\mathbb{T}^{m}\right.$ $\left.\bar{B}_{x_{1}}((1-\eta) \epsilon)\right)$, with a corresponding orthonormal set of eigenfunctions $\left\{\psi_{j,(1-\eta) \epsilon}, j=1,2, \cdots\right\}$. Then

$$
\mathbb{P}_{x}\left[B_{x_{1}}((1-\eta) \epsilon) \cap \beta[0, s]=\emptyset\right]=\sum_{j=1}^{\infty} e^{-s \mu_{j,(1-\eta) \epsilon}} \psi_{j,(1-\eta) \epsilon}(x) \int_{\left.\mathbb{T}^{m} \backslash \bar{B}_{x_{1}}((1-\eta) \epsilon)\right)} d y \psi_{j,(1-\eta) \epsilon}(y) .
$$

Hence, by Parseval's identity and (3.4),

$$
\begin{aligned}
\int_{\mathbb{T}^{m}} d x \mathbb{P}_{x}\left[B_{x_{1}}((1-\eta) \epsilon) \cap \beta[0, s]=\emptyset\right] & =\sum_{j=1}^{\infty} e^{-s \mu_{j,(1-\eta) \epsilon}}\left(\int_{\left.\mathbb{T}^{m} \backslash \bar{B}_{x_{1}}((1-\eta) \epsilon)\right)} \psi_{j,(1-\eta) \epsilon}\right)^{2} \\
& \leq e^{-s \mu_{1,(1-\eta) \epsilon}} \sum_{j=1}^{\infty}\left(\int_{\left.\mathbb{T}^{m} \backslash \bar{B}_{x_{1}}((1-\eta) \epsilon)\right)} \psi_{j,(1-\eta) \epsilon}\right)^{2} \\
& =e^{-s \mu_{1,(1-\eta) \epsilon}}\left(\left|\mathbb{T}^{m}\right|-\left|\bar{B}_{x_{1}}((1-\eta) \epsilon)\right|\right) \\
& \leq e^{-s \mu_{1,(1-\eta) \epsilon}}
\end{aligned}
$$

By (3.3) and (3.5),

$$
\mathbb{P}_{0}\left[T_{\epsilon}>s\right] \leq\left(N^{m} e^{-s \mu_{1,(1-\eta) \epsilon}}\right) \wedge 1 .
$$

Since $\operatorname{diam}\left(\mathbb{T}^{m}\right)=2^{-1} m^{1 / 2}$, the inradius is bounded from above by $4^{-1} m^{1 / 2}$. Moreover, $\{\rho(s)>\epsilon\}=$ $\left\{T_{\epsilon}>s\right\}$. Hence

$$
\mathbb{E}_{0}(\rho(s))=\int_{0}^{4^{-1} m^{1 / 2}} d \epsilon \mathbb{P}_{0}\left[T_{\epsilon}>s\right] \leq \int_{0}^{4^{-1} m^{1 / 2}} d \epsilon\left(\left(N^{m} e^{-s \mu_{1,(1-\eta) \epsilon}}\right) \wedge 1\right) .
$$

Let

$$
N=\left[m^{1 / 2} /(2 \epsilon \eta)\right]+1 .
$$

Since $\epsilon \leq 4^{-1} m^{1 / 2}$ and $\eta<1 / 4$, we have

$$
N \leq m^{1 / 2} /(\epsilon \eta)
$$

- First consider the case $m=2$. By [15], we have that

$$
\mu_{1, \epsilon}=2 \pi(\log (1 / \epsilon))^{-1}+O\left((\log (1 / \epsilon))^{-2}\right), \quad \epsilon \downarrow 0 .
$$

Hence there exists $\epsilon_{0}(\eta)$ such that, for $\epsilon \leq \epsilon_{0}(\eta)$,

$$
\mu_{1, \epsilon} \geq 2 \pi(\log (1 / \epsilon))^{-1}(1-\eta) .
$$

So, abbreviating $\epsilon \leq \epsilon_{1}(\eta)=\left(2^{1 / 2} / 4\right) \wedge \epsilon_{0}(\eta)$, we have for $\epsilon \leq \epsilon_{1}(\eta)$,

$$
\begin{aligned}
\mu_{1,(1-\eta) \epsilon} & \geq 2 \pi(\log (1 /(1-\eta) \epsilon))^{-1} \\
& =2 \pi(\log (1 / \epsilon))^{-1}\left(1+\frac{\log (1 /(1-\eta))}{\log (1 / \epsilon)}\right)^{-1}(1-\eta) \\
& \geq 2 \pi(\log (1 / \epsilon))^{-1}\left(1+\frac{2 \log (1 /(1-\eta))}{3 \log 2}\right)^{-1}(1-\eta) \\
& \geq 2 \pi(\log (1 / \epsilon))^{-1}(1-\log (1 /(1-\eta)))(1-\eta) \\
& \geq 2 \pi(\log (1 / \epsilon))^{-1}\left(1-\frac{\eta}{1-\eta}\right)(1-\eta) \\
& =2 \pi(\log (1 / \epsilon))^{-1}(1-2 \eta) .
\end{aligned}
$$


Putting (3.6), (3.8) and (3.9) together, we obtain

$$
\begin{array}{r}
\mathbb{E}_{0}(\rho(s)) \leq \int_{0}^{\epsilon_{1}(\eta)} d \epsilon\left(\left(2(\eta \epsilon)^{-2} e^{-2 \pi s(\log (1 / \epsilon))^{-1}(1-2 \eta)}\right) \wedge 1\right) \\
+2 \int_{\epsilon_{1}(\eta)}^{\infty} d \epsilon(\eta \epsilon)^{-2} e^{-2 \pi s\left(\log \left(1 / \epsilon_{1}(\eta)\right)\right)^{-1}(1-2 \eta)}
\end{array}
$$

The second term in (3.10) is bounded from above by

$$
2 \eta^{-2} \epsilon_{1}(\eta)^{-1} e^{-(\pi / 2) s\left(\log \left(1 / \epsilon_{1}(\eta)\right)\right)^{-1}}
$$

By changing variables, $\epsilon=e^{-\theta}$, we obtain that the first integral is bounded from above by

$$
\begin{aligned}
& 2 \eta^{-2} \int_{0}^{\infty} d \theta e^{-\theta}\left(e^{2 \theta-2 \pi s(1-2 \eta) / \theta} \wedge 1\right) \\
& \leq 2 \eta^{-2} \int_{0}^{(\pi s(1-2 \eta))^{1 / 2}} d \theta e^{\theta-2 \pi s(1-2 \eta) / \theta}+2 \eta^{-2} \int_{(\pi s(1-2 \eta))^{1 / 2}}^{\infty} d \theta e^{-\theta} \\
& \leq 2 \eta^{-2}\left((\pi s)^{1 / 2}+1\right) e^{-(\pi s(1-2 \eta))^{1 / 2}} .
\end{aligned}
$$

It follows from (3.10 3.11) that for $\eta \in(0,1 / 4)$,

$$
\limsup _{s \rightarrow \infty} s^{-1 / 2} \log \mathbb{E}_{0}(\rho(s)) \leq-(\pi(1-2 \eta))^{1 / 2} .
$$

This proves the upper bound in Theorem 1.3 for $m=2$ because $\eta \in(0,1 / 4)$ was arbitrary.

- Next consider the case $m=3,4, \cdots$. By Theorem 1 in [8], we have that

$$
\mu_{1, \epsilon}=\kappa_{m} \epsilon^{m-2}(1+o(1)), \quad \epsilon \downarrow 0 .
$$

Hence there exists $\epsilon_{0}(\eta)$ such that, for $\epsilon \leq \epsilon_{0}(\eta)$,

$$
\mu_{1, \epsilon} \geq \kappa_{m} \epsilon^{m-2}(1-\eta)
$$

By (3.6), (3.8) and (3.12) we have, for any $\eta \in(0,1 / 4)$,

$$
\begin{aligned}
\mathbb{E}_{0}(\rho(s)) \leq & \int_{0}^{\min \left\{m^{1 / 2} / 4, \epsilon_{0}(\eta)\right\}} d \epsilon\left(m^{m / 2}(\epsilon \eta)^{-m} e^{-s \kappa_{m}(1-\eta) \epsilon^{m-2}} \wedge 1\right) \\
& +\int_{\min \left\{m^{1 / 2} / 4, \epsilon_{0}(\eta)\right\}}^{m^{1 / 2} / 4} d \epsilon m^{m / 2}(\epsilon \eta)^{-m} e^{-s \kappa_{m}(1-\eta) \epsilon_{0}(\eta)^{m-2}} .
\end{aligned}
$$

The second term in (3.13) is bounded from above by

$$
m^{m / 2}(m-1)^{-1} \eta^{-m}\left(\max \left\{4 / m^{1 / 2}, \epsilon_{0}(\eta)^{-1}\right\}\right)^{m-1} e^{-s \kappa_{m}(1-\eta) \epsilon_{0}(\eta)^{m-2}} .
$$

The first term in (3.13) is bounded from above by

$$
\begin{aligned}
\int_{0}^{\infty} & d \epsilon\left(m^{m / 2}(\epsilon \eta)^{-m} e^{-s \kappa_{m}(1-\eta) \epsilon^{m-2}} \wedge 1\right) \\
& =(1-\eta)^{-1 /(m-2)}(m-2)^{-1}\left(s \kappa_{m}\right)^{-1 /(m-2)} \int_{0}^{\infty} d \theta \theta^{(3-m) /(m-2)}\left(K \theta^{-m /(m-2)} e^{-\theta} \wedge 1\right),
\end{aligned}
$$

where

$$
K=m^{m / 2} \eta^{-m}(1-\eta)^{m /(m-2)}\left(s \kappa_{m}\right)^{m /(m-2)} .
$$

Let $\theta_{K}$ be the unique positive root of

$$
K \theta^{-m /(m-2)} e^{-\theta}=1 .
$$


The integral in the right-hand side of (3.15) equals

$$
\begin{array}{rl}
\int_{0}^{\theta_{K}} & d \theta \theta^{(3-m) /(m-2)}+K \int_{\theta_{K}}^{\infty} d \theta \theta^{(3-2 m) /(m-2)} e^{-\theta} \\
& \leq(m-2) \theta_{K}^{1 /(m-2)}+K \theta_{K}^{(3-2 m) /(m-2)} e^{-\theta_{K}} \\
& =(m-2) \theta_{K}^{1 /(m-2)}+\theta_{K}^{(3-m) /(m-2)} .
\end{array}
$$

For $K \geq e$, we have $\theta_{K} \geq 1$, and, by (3.17),

$$
e^{\theta_{K}}=K \theta_{K}^{-m /(m-2)} \leq K, \quad K \geq e .
$$

Hence $\theta_{K} \leq \log K$ for $K \geq e$. It follows from (3.13(3.19) that for $s \rightarrow \infty$,

$$
\mathbb{E}_{0}(\rho(s)) \leq(1-\eta)^{-1 /(m-2)}(\log K)^{1 /(m-2)}\left(s \kappa_{m}\right)^{-1 /(m-2)}+O\left(s^{-1 /(m-2)}(\log s)^{(3-m) /(m-2)}\right) .
$$

Hence

$$
\limsup _{s \rightarrow \infty}\left(\frac{s}{\log s}\right)^{1 /(m-2)} \mathbb{E}_{0}(\rho(s)) \leq(1-\eta)^{-1 /(m-2)}\left(\frac{m}{(m-2) \kappa_{m}}\right)^{1 /(m-2)} .
$$

Let $\eta \downarrow 0$ to get the upper bound in Theorem 1.2 .

\subsection{Lower bound for $m \geq 3$}

To prove the lower bound in Theorem 1.2 we use the following inequality in [9]. Let $\eta \in(0,1 / 10]$ and $\delta \in(0,1 / 10]$ be arbitrary, and let, for $n \in \mathbb{N}$,

$$
\begin{gathered}
\epsilon_{n}=(1-\eta)^{n}, \\
v_{n}=(1-\eta) \kappa_{m}^{-1} \epsilon_{n}^{2-m},
\end{gathered}
$$

and

$$
K_{n} \geq \epsilon_{n}^{-m(1-2 \delta)}
$$

The very last inequality of 9 implies that for the sequence $\left(\epsilon_{n}\right)$ there exists $c=c(\eta, \delta)$ such that

$$
\mathbb{P}_{0}\left[T_{\epsilon_{n}} \leq(1-2 \eta) v_{n} \log K_{n}\right] \leq 4(1-\eta)^{c n} .
$$

Let $\phi \in(0,1 / 4]$ be arbitrary. There exists $N(\eta, \delta, \phi) \in \mathbb{N}$ such that for $n \geq N(\eta, \delta, \phi)$,

$$
\mathbb{P}_{0}\left[T_{\epsilon_{n}} \leq(1-2 \eta) v_{n} \log K_{n}\right] \leq \phi .
$$

or

$$
\mathbb{P}_{0}\left[T_{\epsilon_{n}} \geq(1-2 \eta) v_{n} \log K_{n}\right] \geq 1-\phi .
$$

This, together with (3.21 3.22 , gives that

$$
\mathbb{P}_{0}\left[T_{\epsilon_{n}} \geq C m \kappa_{m}^{-1} \epsilon_{n}^{2-m} \log \left(\epsilon_{n}^{-1}\right)\right] \geq 1-\phi,
$$

where $C=(1-2 \eta)(1-\eta)(1-2 \delta)$. We now choose $n=n(s, \eta) \in \mathbb{Z}$ such that

$$
(1-\eta)^{n-1} \geq\left(C(m-2)^{-1} m \kappa_{m}^{-1} \frac{\log s}{s}\right)^{1 /(m-2)} \geq(1-\eta)^{n} .
$$

Then $n \in \mathbb{N}$ and $n \geq N(\eta, \delta, \phi)$ for all $s$ large enough. By (3.20) and (3.24)

$$
\epsilon_{n}^{2-m}=(1-\eta)^{n(2-m)} \geq C^{-1}(m-2) m^{-1} \kappa_{m} \frac{s}{\log s} .
$$


On the other hand, by (3.20) and (3.24) we have that

$$
\log \left(\epsilon_{n}^{-1}\right) \geq(m-2)^{-1} \log \left(C^{-1}(m-2) m^{-1} \kappa_{m} \frac{s}{\log s}\right) .
$$

By (3.25 3.26),

$$
C m \kappa_{m}^{-1} \epsilon_{n}^{2-m} \log \left(\epsilon_{n}^{-1}\right) \geq s+h(s),
$$

where

$$
h(s)=\frac{s}{\log s} \log \left(\frac{(m-2) m^{-1} \kappa_{m}}{\log s}\right) .
$$

By the definition of $n$ in (3.24) and by (3.23) and (3.27), we have that for all $s$ sufficiently large,

$$
\mathbb{P}_{0}\left[T_{\epsilon_{n}} \geq s+h(s)\right] \geq 1-\phi .
$$

Hence, by the first equality in (3.7), (3.20) and (3.24),

$$
\begin{aligned}
\mathbb{E}_{0}(\rho(s+h(s))) & =\int_{0}^{4^{-1} m^{1 / 2}} d \epsilon \mathbb{P}_{0}\left[T_{\epsilon}>s+h(s)\right] \\
& \geq \int_{0}^{\epsilon_{n}} d \epsilon \mathbb{P}_{0}\left[T_{\epsilon}>s+h(s)\right] \\
& \geq(1-\phi) \epsilon_{n} \\
& \geq(1-\phi)(1-\eta)\left(C(m-2)^{-1} m \kappa_{m}^{-1} \frac{\log s}{s}\right)^{1 /(m-2)} .
\end{aligned}
$$

It remains to show that (3.28) implies the lower bound in Theorem 1.2 We abbreviate $\sigma=s+h(s)$. Since $(\log \log s) / \log s \rightarrow 0$ as $s \rightarrow \infty$, we have that $|h(s)| \leq s / 2$ for all $s$ sufficiently large. Hence $\sigma \geq s / 2$ for all such $s$, and $s=\sigma-h(s) \leq \sigma-h(2 \sigma)$. It follows that for all such $s$,

$$
\mathbb{E}_{0}(\rho(\sigma)) \geq(1-\phi)(1-\eta)\left(C(m-2)^{-1} m \kappa_{m}^{-1} \frac{\log \sigma}{\sigma-h(2 \sigma)}\right)^{1 /(m-2)} .
$$

In particular, it follows that

$$
\begin{aligned}
\liminf _{\sigma \rightarrow \infty} & \mathbb{E}_{0}(\rho(\sigma))\left(\frac{\sigma}{\log \sigma}\right)^{1 /(m-2)} \\
& \geq(1-\phi)(1-\eta)\left(C(m-2)^{-1} m \kappa_{m}^{-1} \liminf _{\sigma \rightarrow \infty} \frac{\sigma}{\sigma-h(2 \sigma)}\right)^{1 /(m-2)} \\
& =(1-\phi)(1-\eta)\left(C(m-2)^{-1} m \kappa_{m}^{-1}\right)^{1 /(m-2)} .
\end{aligned}
$$

Letting first $\phi \downarrow 0$, then $\delta \downarrow 0$ and finally $\eta \downarrow 0$, we conclude from (3.29) that

$$
\liminf _{\sigma \rightarrow \infty} \mathbb{E}_{0}(\rho(\sigma))\left(\frac{\sigma}{\log \sigma}\right)^{1 /(m-2)} \geq\left((m-2)^{-1} m \kappa_{m}^{-1}\right)^{1 /(m-2)} .
$$

This proves the lower bound in Theorem 1.2 .

\subsection{Lower bound for $m=2$}

To prove the lower bound in Theorem 1.3 we use the following inequality in [10]. Let $\delta \in(0,1 / 10]$ be arbitrary, fix $\gamma \in(0,1-\delta)$ and let

$$
\epsilon_{n}=2 n^{\gamma-1} \text {. }
$$


It was shown in [10] that there exist $N_{0}(\gamma, \delta) \in \mathbb{N}$ such that for all $n \geq N_{0}(\gamma, \delta)$,

$$
\mathbb{P}_{0}\left[T_{\epsilon_{n}} \geq \pi^{-1}(1-\gamma-\delta)^{2}(\log n)^{2}\right] \geq 1-\delta .
$$

We let $n=n(s, \gamma, \delta) \in\{2,3, \cdots\}$ be such that

$$
\pi^{-1}(1-\gamma-\delta)^{2}(\log n)^{2} \geq s \geq \pi^{-1}(1-\gamma-\delta)^{2}(\log (n-1))^{2} .
$$

It follows that, for all $s$ sufficiently large and $n \geq N_{0}(\gamma, \delta)$,

$$
\mathbb{P}_{0}\left[T_{\epsilon_{n}} \geq s\right] \geq 1-\delta
$$

In particular, for all $s$ sufficiently large we have that

$$
\begin{aligned}
\mathbb{E}_{0}(\rho(s)) & =\int_{0}^{4^{-1} \sqrt{2}} d \epsilon \mathbb{P}_{0}\left[T_{\epsilon} \geq s\right] \geq \int_{0}^{\epsilon_{n}} d \epsilon \mathbb{P}_{0}\left[T_{\epsilon} \geq s\right] \\
& \geq \int_{0}^{\epsilon_{n}} d \epsilon \mathbb{P}_{0}\left[T_{\epsilon_{n}} \geq s\right] \geq \epsilon_{n}(1-\delta)=2 n^{\gamma-1}(1-\delta) .
\end{aligned}
$$

By the second inequality in (3.30), we have that

$$
n \leq 1+e^{(\pi s)^{1 / 2} /(1-\gamma-\delta)} .
$$

Since $1 \leq e^{(\pi s)^{1 / 2} /(1-\gamma-\delta)}$, we have by 3.313.32 that

$$
\mathbb{E}_{0}(\rho(s)) \geq 2^{\gamma}(1-\delta) e^{(\gamma-1)(\pi s)^{1 / 2} /(1-\gamma-\delta)} .
$$

Hence

$$
\liminf _{s \rightarrow \infty} s^{-1 / 2} \log \mathbb{E}_{0}(\rho(s)) \geq(\gamma-1) \pi^{1 / 2} /(1-\gamma-\delta)
$$

Letting $\delta \downarrow 0$ we obtain from (3.33) that

$$
\liminf _{s \rightarrow \infty} s^{-1 / 2} \log \mathbb{E}_{0}(\rho(s)) \geq-\pi^{-1 / 2} .
$$

This proves the lower bound in Theorem 1.3 .

\section{References}

[1] A. Ancona, On strong barriers and an inequality of Hardy for domains in $\mathbb{R}^{n}$, J. Lond. Math. Soc. 34 (1986) 274-290.

[2] M. van den Berg, Heat content and Brownian motion for some regions with a fractal boundary, Probab. Theor. Rel. Fields 100 (1994) 439-456.

[3] M. van den Berg, Heat equation on the arithmetic von Koch snowflake, Probab. Theor. Rel. Fields 118 (2000) 17-36.

[4] M. van den Berg, E. Bolthausen, F. den Hollander, Brownian survival among Poissonian traps with random shapes at critical intensity, Probab. Theor. Rel. Fields 132 (2005) 163-202.

[5] M. van den Berg, P. B. Gilkey, Heat content asymptotics of a Riemannian manifold with boundary, J. Funct. Anal. 120 (1994) 48-71.

[6] M. van den Berg, F. den Hollander, Asymptotics for the heat content of a planar region with a fractal polygonal boundary, Proc. Lond. Math. Soc. 78 (1999) 627-661.

[7] M. van den Berg, J.-F. Le Gall, Mean curvature and the heat equation, Math. Zeit. 215 (1994) 437-464. 
[8] I. Chavel, E. A. Feldman, Spectra of manifolds less a small domain, Duke Mathematical J. 56 (1988) 399-414.

[9] A. Dembo, Y. Peres, J. Rosen, Brownian motion on compact manifolds: cover time and late points, Elect. J. Probab. 8 (2003) 1-14.

[10] A. Dembo, Y. Peres, J. Rosen, O. Zeitouni, Cover times for Brownian motion and random walks in two dimensions, Ann. Math. 160 (2004) 433-464.

[11] P. B. Gilkey, Asymptotic Formulae in Spectral Geometry, Studies in Mathematics, Chapman \& Hall/CRC, Boca Raton, Fl, 2004.

[12] J.-F. Le Gall, Sur une conjecture de M. Kac, Probab. Theor. Rel. Fields 78 (1988) 389-402.

[13] J.-F. Le Gall, Wiener sausage and self-intersection local times, J. Funct. Anal. 88 (1990) 299-341.

[14] J. Goodman and F. den Hollander, Extremal geometry of a Brownian porous medium. arXiv:1211.3630 $1[$ math.PR]

[15] S. Ozawa, The first eigenvalue of the Laplacian on two-dimensional Riemannian manifolds, Tôhoku Math. J. 34 (1982) 7-14.

[16] S. C. Port, Spitzer's formula involving capacity, in: Random Walks, Brownian Motion, and Interacting Particle Systems (eds. R. Durrett, H. Kesten), Progress in Probability 28, Birkhäuser, Boston, MA, 1991.

[17] M. Reed, B. Simon, Methods of Modern Mathematical Physics IV, Analysis of Operators, Academic Press, New York, NY, 1978.

[18] F. Spitzer, Electrostatic capacity, heat flow and Brownian motion, Z. Wahrscheinlichkeitstheorie Verw. Gebiete 3 (1964) 187-197.

[19] F. Spitzer, Principles of Random Walk, D. Van Nostrand Co. Inc., Princeton, NJ, 1964.

[20] A.-S. Sznitman, Brownian Motion, Obstacles and Random Media, Springer, Berlin, 1998. 